\title{
Oridonin effectively reverses cisplatin drug resistance in human ovarian cancer cells via induction of cell apoptosis and inhibition of matrix metalloproteinase expression
}

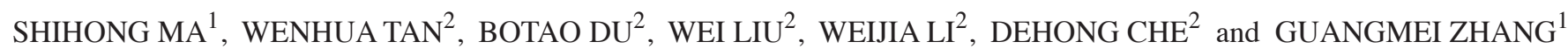 \\ ${ }^{1}$ Department of Gynecology and Obstetrics, The First Affiliated Hospital of Harbin Medical University, \\ Harbin, Heilongjiang $150001 ;^{2}$ Department of Gynecology and Obstetrics, \\ The Second Affiliated Hospital of Harbin Medical University, Harbin, Heilongjiang 150086, P.R. China
}

Received March 13, 2015; Accepted January 11, 2016

DOI: $10.3892 / \mathrm{mmr} .2016 .4897$

\begin{abstract}
Cisplatin is a first generation platinum-based chemotherapeutic agent, however, the extensive application of cisplatin inevitably results in drug resistance, which is a major obstacle in cancer chemotherapy. The aim of the present study was to investigate the efficiency of reversing cisplatin-resistance with the use of combination therapy with oridonin and cisplatin in human ovarian cancer cells, and attempt to reduce the side effects of the therapeutic agents when used alone. The half maximal inhibitory concentration $\left(\mathrm{IC}_{50}\right)$ values of cisplatin were determined in cisplatin-sensitive and cisplatin-resistant ovarian cancer cells using an MTT assay. $\mathrm{IC}_{50}$ values of cisplatin in A2780, A2780/DDP, SKOV3 and SKOV3/DDP cells were significantly decreased in a time-dependent manner. The antitumor effect of oridonin in A2780/DDP cells was also detected by the MTT assay and the inhibitory effects of oridonin increased in a dose- and time-dependent manner. A2780/DDP cells were treated with $20 \mu \mathrm{M}$ oridonin in combination with increasing concentrations of cisplatin for $48 \mathrm{~h}$, and the result demonstrated that oridonin synergistically increased the antitumor effects of cisplatin in A2780/DDP cells. Notably, the combination treatment of oridonin and cisplatin effectively reversed cisplatin resistance and the $\mathrm{IC}_{50}$ values were significantly decreased from $50.97 \mu \mathrm{M}$ and 135.20 to $26.12 \mu \mathrm{M}$ and $73.00 \mu \mathrm{M}$ in A2780/DDP and SKOV3/DDP cells at $48 \mathrm{~h}$, respectively. Furthermore, oridonin induced cell apoptosis in a dose-dependent manner and promoted cell-cycle arrest at the $\mathrm{G}_{0} / \mathrm{G}_{1}$ phase in ovarian cancer cells. Oridonin and cisplatin synergistically increased the cell
\end{abstract}

Correspondence to: Dr Guangmei Zhang, Department of Gynecology and Obstetrics, The First Affiliated Hospital of Harbin Medical University, 199 Dongdazhi Street, Nangang, Harbin, Heilongjiang 150001, P.R. China

E-mail: zhangguangmeiwork@163.com

Key words: oridonin, cisplatin, ovarian cancer, drug resistance, cell apoptosis apoptosis rate of A2780/DDP cells, which was detected by fluorescence-activated cell sorting analysis. Downregulated expression levels of $\mathrm{Bcl}-2$ and upregulated the expression of Bax protein were demonstrated by western blot analysis, further indicating increased apoptosis. In addition, the expression levels of matrix metalloproteinase (MMP)-2 and MMP-9 decreased in a dose-dependent manner with oridonin treatment. The results from the present study demonstrated that oridonin exerted a synergistic effect with cisplatin to inhibit proliferation and induce cell apoptosis in cisplatin-resistant ovarian cancer cells. Thus, combination therapy with oridonin and cisplatin effectively reversed cisplatin resistance in human ovarian cancer cells, which may have useful clinical applications.

\section{Introduction}

Ovarian cancer has the lowest survival rate of all gynecological malignancies (1). Ovarian cancers predominantly include three types of tumor, epithelial tumors, germ cell tumors and stromal tumors (2). Of all ovarian cancers, $85-90 \%$ are epithelial ovarian carcinomas $(3,4)$ and $~ 70 \%$ of patients are diagnosed at an advanced stage with pelvic or lymph node metastasis. Only $1 / 4$ patients are diagnosed at an early stage and the worldwide five-year survival rate of patients with advanced ovarian cancer is $20-25 \%(5,6)$. It is difficult to detect ovarian cancer at an early stage predominantly due to its inherent metastatic nature resulting in a poor prognosis (7).

Surgery combined with chemotherapy is an important therapeutic strategy for ovarian cancer. Cisplatin is a first line platinum-based chemotherapeutic agent, which exerts marked antitumor activity in a number of solid tumors (8-12). It is also one of the most commonly used agents for the treatment of ovarian cancer. However, the extensive application of cisplatin may result in adverse gastrointestinal toxicity, including severe nausea and vomiting, renal toxicity and neurotoxicity (13). Notably, long-term use of cisplatin results in drug resistance, which is a major obstacle in cancer chemotherapy (14-16). Investigation into therapeutic strategies with increased efficacy in order to decrease the side effects of treatment or reduce drug resistance in ovarian cancer is required. 
Recently, natural products extracted from medicinal plants have drawn more attention in cancer therapy. Oridonin is extracted from the Chinese herb Rabdosia rubescens, and is a natural compound with the structure of a tetracycline diterpenoid $(17,18)$. It has been reported to exert antitumor effects and is widely used, in China, in the clinical treatment of a number of tumor types. Qi et al (19) reported that oridonin effectively induced cell apoptosis of pancreatic cancer cells, and a nanosuspension was more effective than free oridonin on $\mathrm{G}_{2} / \mathrm{M}$-phase cell cycle arrest and apoptosis in the PANC-1 human pancreatic cancer cell line. Gao et al (20) demonstrated that oridonin induces apoptosis and senescence by increasing hydrogen peroxide and glutathione depletion in colorectal cancer cells. Furthermore, a study demonstrated that autophagy preceded apoptosis in oridonin-treated MCF-7 human breast cancer cells (21). In lung cancer patients, oridonin also suppressed mammalian target of rapamycin (mTOR) signaling and the growth of lung cancer tumors, suggesting inhibition of mTORC1 may be an effective target for improving the therapeutic outcome of treatment with oridonin (22).

In the present study, two cisplatin-resistant ovarian cancer cell lines, A2780/DDP and SKOV3/DDP, were used to investigate the underlying mechanism of combined therapy with oridonin and cisplatin. It is beneficial to elucidate the molecular mechanism of disease progression to aid in the development of therapeutic strategies that reverse drug resistance.

\section{Materials and methods}

Therapeutic agents and cell lines. Oridonin and cisplatin were obtained from the Shifeng Biocorporation (Shanghai, China). 3-(4,5-dimethylthiazol-2-yl)-2,5-diphenyltetrazolium bromide (MTT) was purchased from Sigma-Aldrich (St. Louis, MO, USA). The A2780/DDP cisplatin-resistant human ovarian cancer subline (Huiying Corporation, Shanghai, China) and SKOV3/DDP (Yunnan Tumor Hospital, Kunming, China) were used. The non-resistant cells lines, A2780 and SKOV3 were obtained from Wuhan Boster Biological Technology, Ltd. (Wuhan, China). The cells were cultured in Dulbecco's modified Eagle's medium (Hyclone; GE Healthcare Life Sciences, Logan, UT, USA) enriched with $10 \%$ fetal bovine serum (Hyclone; GE Healthcare Life Sciences) at $37^{\circ} \mathrm{C}$ and $5 \% \mathrm{CO}_{2}$. Cisplatin was obtained from Qilu Pharmaceutical Co., Ltd. (Jinan, China) and $0.5 \mu \mathrm{g} / \mathrm{ml}$ cisplatin was added into the medium to maintain chemoresistance in the resistant cells.

MTT assay. The inhibitory effect of oridonin alone, cisplatin alone and oridonin + cisplatin on A2780/DDP and SKOV3/DDP human ovarian cancer cells was measured using the MTT assay. Cells were transferred to cisplatin-free medium 3 days prior to the experiments. The cells (1.0x $10^{4}$ cells/well) were plated into 96 -well plates and allowed to attach overnight. The cells were treated with different concentrations of oridonin $(10,40,80$ and $160 \mu \mathrm{mol} / \mathrm{l})$ or cisplatin $(1,2,5,10,20,40,80,200,500$ or $1,000 \mu \mathrm{M})$ for 24, 48 and 72 h, respectively. Control cells were administered an equal quantity of dimethyl sulfoxide (DMSO). MTT $(20 \mu \mathrm{l} ; 5 \mathrm{mg} / \mathrm{ml})$ was added to each well and incubated for $4 \mathrm{~h}$ at $37^{\circ} \mathrm{C}$ in the dark. The supernatant was removed and the formazan crystals were dissolved in $100 \mu 1 \mathrm{DMSO}$ and mixed thoroughly prior to determining absorbance at a wavelength of $490 \mathrm{~nm}$ using an AquaMate-Plus ultraviolet spectrophotometer (Thermo Fisher Scientific, Inc.). All in vitro experiments were conducted in triplicate.

Apoptosis rate analysis. Flow cytometry was used to detect the apoptosis rate. A2780/DDP cells $\left(2 \times 10^{4}\right.$ cells/well) were plated into 6 -well plates. They were cultured for 6 to $8 \mathrm{~h}$ and treated with cisplatin alone, oridonin alone, or cisplatin in combination of oridonin for $48 \mathrm{~h}$. The oridonin concentrations used were: $0,10,20,40,80$ and $160 \mu \mathrm{mol} / 1$ and $50 \mu \mathrm{M}$ cisplatin. The cells were analyzed following treatment with RNase (Sigma-Aldrich) and stained with Annexin V and propidium iodide (PI; Sigma-Aldrich) for flow cytometry (BD FACSCalibur $^{\mathrm{TM}}$; BD Biosciences, Franklin Lakes, NJ, USA).

Western blotting. Western blotting was performed as described previously (23-25). Briefly, a total protein extract for each tissue sample or cell line was dissolved in lysis buffer and equal quantities of protein $(60 \mu \mathrm{g})$ were analyzed by immunoblotting. Rabbit anti-human polyclonal antibodies against Bcl-2 (cat. no. sc-492), rabbit anti-human polyclonal antibodies against Bax (cat. no. 526), rabbit anti-human polyclonal antibodies against MMP-2 (cat. no. sc-10736) and goat anti-human polyclonal IgG antibodies against MMP-9 (cat. no. sc-6840) were purchased from Santa Cruz Biotechnology Inc. (Dallas, TX, USA) and used at a dilution of 1:1,000. The horseradish peroxidase-conjugated goat anti-rabbit secondary antibody was obtained from Abgent, Inc. (San Diego, CA, USA; cat. no. ASS1006).

Statistical analysis. All data were processed using SPSS 17.0 (SPSS, Inc., Chicago, IL, USA). Statistical analysis was performed using analysis of variance and Student's t-test for continuous data. The data are presented as the mean \pm standard error of the mean and $\mathrm{P}<0.05$ was considered to indicate a statistically significant difference.

\section{Results}

Half maximal inhibitory concentration $\left(I C_{50}\right)$ value of cisplatin in ovarian cancer cells. In order to detect the antitumor effects of cisplatin on ovarian cancer cells, the A2780 and SKOV3 human ovarian cancer cell lines and the cisplatin-resistant sublines, A2780/DDP and SKVO3/DDP, were used as cell models. The ovarian cancer cells were treated with increasing concentrations of cisplatin and the inhibitory rate was determined by an MTT assay. The structure of oridonin and cisplatin is presented in Fig. 1. Cisplatin had an increasing antitumor effect in A2780 and A2780/DDP human ovarian cancer cell lines in a dose- and time-dependent manner. As presented in Fig. 2, the $\mathrm{IC}_{50}$ values of cisplatin were $88.89,13.20$ and $9.55 \mu \mathrm{M}$ for 24,48 and $72 \mathrm{~h}$ in the $\mathrm{A} 2780$ sensitive cell line, respectively. However, the $\mathrm{IC}_{50}$ values were $350.50,50.96$ and $25.39 \mu \mathrm{M}$ in the A2780/DDP cisplatin-resistant cell line following treatment for 24, 48 and $72 \mathrm{~h}$, respectively. $\mathrm{IC}_{50}$ values in SKOV3 and SKOV3/DDP were $105.10,51.73,16.13$ and $446.70,135.00,66.70 \mu \mathrm{M}$, respectively, for 24, 48 and $72 \mathrm{~h}$. 
A

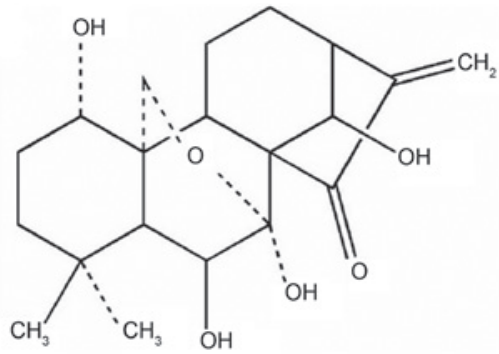

B<smiles>N[Pb](N)(Cl)Cl</smiles>

Figure 1. Chemical structures of (A) oridonin and (B) cisplatin.

A2780

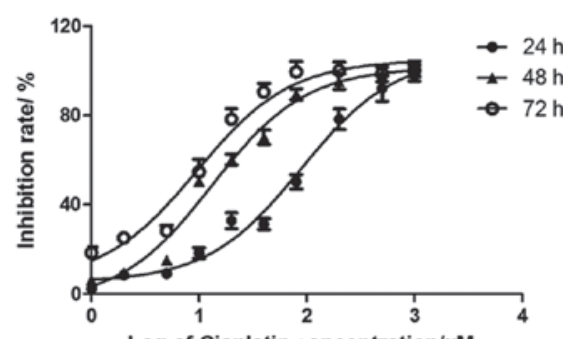

Log of Cisplatin concentration/ $/ \mathrm{M}$

\begin{tabular}{|c|c|c|c|}
\hline & $24 \mathrm{~h}$ & $48 \mathrm{~h}$ & $72 \mathrm{~h}$ \\
\hline$I \mathrm{C} 50$ & 88.89 & 13.20 & 9.55 \\
\hline
\end{tabular}

SKOV3

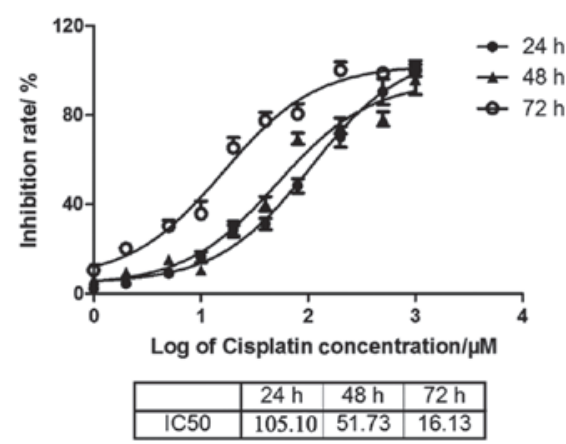

A2780/DDP

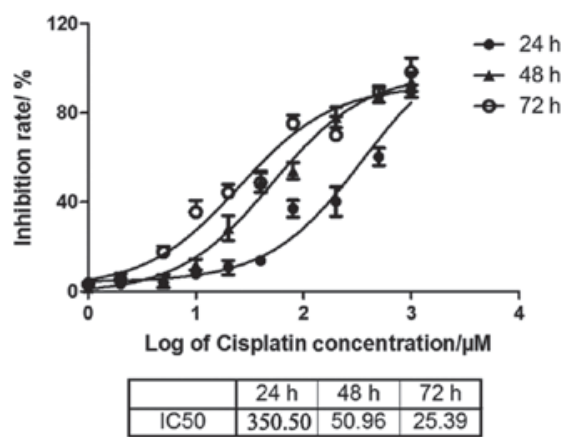

SKVO3/DDP

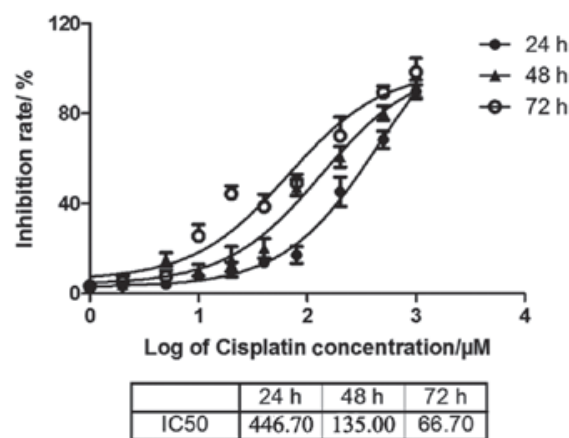

Figure 2. IC $_{50}$ values of cisplatin on ovarian cancer cells are determined by an MTT assay. The A2780, A2780/DDP, SKOV3 and SKOV3/DDP ovarian cancer cell lines ( $3 \times 10^{4}$ cells/per well) were plated into 96 wells and the cells were exposed to increasing concentrations of cisplatin for 24,48 and $72 \mathrm{~h}$. The proliferation of ovarian cancer cells was determined by the MTT assay and $\mathrm{IC}_{50}$ values were calculated. The histogram of $\mathrm{IC}_{50}$ values were presented at 24,48 and $72 \mathrm{~h}$. All the analyses were repeated three times on duplicate samples. $\mathrm{IC}_{50}$, half maximal inhibitory concentration; MTT, 3-(4,5-dimethylthiazol-2-yl)-2,5-diphenyltetrazolium bromide.

A

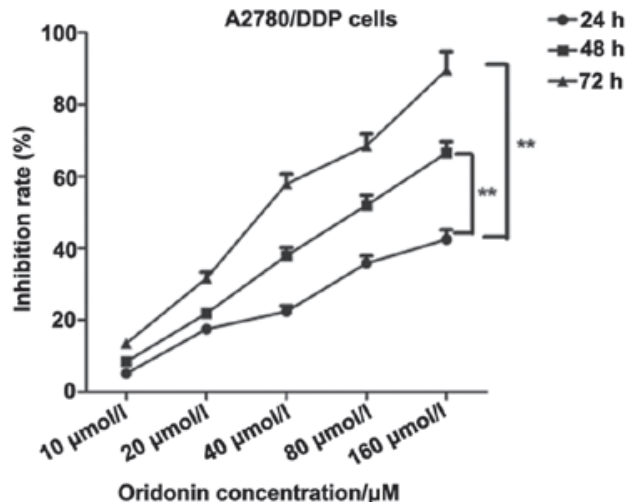

B

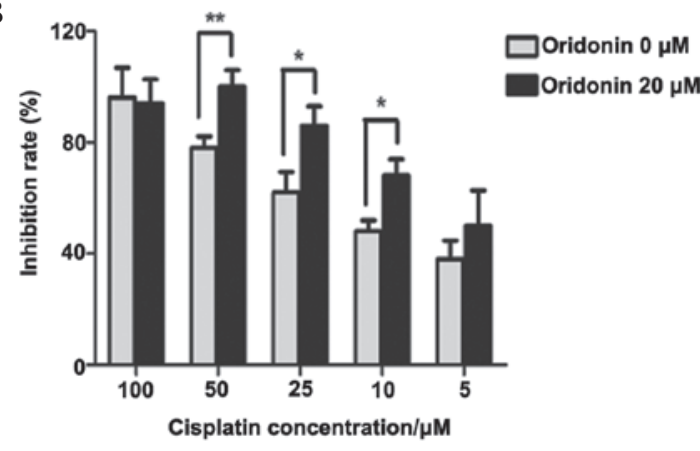

Figure 3. Proliferation of A2780/DDP cells was determined by the MTT assay. (A) The A2780/DDP cisplatin-resistant ovarian cancer cell line (3x10 3 cells/per well) was plated into 96-well plates and treated with increasing concentrations of oridonin for 24,48 and $72 \mathrm{~h}$. Data are presented as the mean \pm standard deviation in triplicates. ${ }^{* *} \mathrm{P}<0.01$ vs. $24 \mathrm{~h}$. (B) A2780/DDP cells were treated with $20 \mu \mathrm{M}$ oridonin in combination with increasing concentrations of cisplatin for $48 \mathrm{~h}$. The inhibition rate was determined by the MTT assay. Cells not treated with oridonin served as controls. " $\mathrm{P}<0.05$ and ${ }^{* *} \mathrm{P}<0.01$ vs. the control. MTT, 3-(4,5-dimethylthiazol-2-yl)-2,5-diphenyltetrazolium bromide. 
A

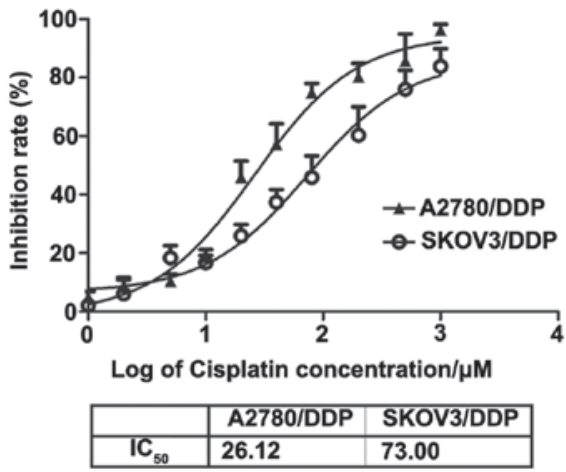

B

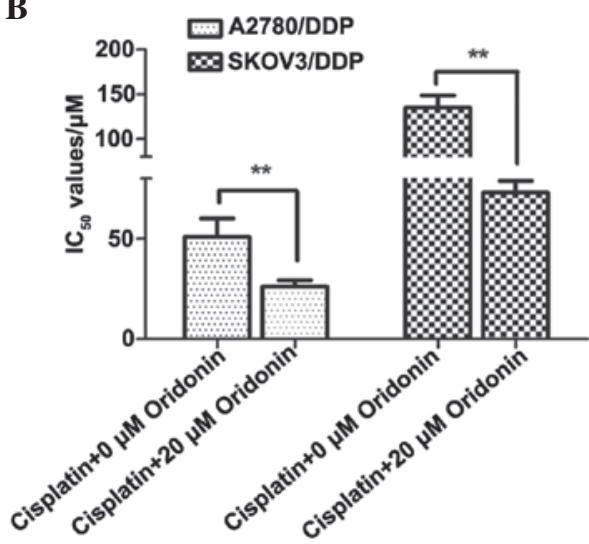

Figure 4. $\mathrm{IC}_{50}$ values of cisplatin were decreased in combination with $20 \mu \mathrm{M}$ oridonin in A2780/DDP and SKOV3/DDP cells. (A) The cisplatin resistant ovarian cancer cells, A2780/DDP and SKOV3/DDP $\left(3 \times 10^{3}\right.$ cells/per well), were plated into 96 -well plates and treated with increasing concentrations of oridonin for $48 \mathrm{~h}$. The MTT assay was performed to detect the proliferation of cisplatin-resistant ovarian cancer cells. IC $_{50}$ values were calculated and the data are presented as the mean \pm standard deviation. Experiments were repeated three times. (B) The histogram of $\mathrm{IC}_{50}$ values in A2780/DDP and SKOV3/DDP cells. The cisplatin-resistant ovarian cancer cells were treated with $20 \mu \mathrm{M}$ oridonin in combination with increasing concentrations of cisplatin for $48 \mathrm{~h}$. The MTT assay was used to detect the inhibition rate of oridonin in combination with cisplatin. The cells treated with cisplatin alone served as controls. ${ }^{* *} \mathrm{P}<0.01$ vs. the control. $\mathrm{IC}_{50}$, half maximal inhibitory concentration; MTT, 3-(4,5-dimethylthiazol-2-yl)-2,5-diphenyltetrazolium bromide.

Oridonin synergistically increases the antitumor effects of cisplatin in A2780/DDP cells. The inhibitory effects of oridonin in ovarian cancer cells were also detected by an MTT assay. The A2780/DDP cisplatin resistant ovarian cancer cells were treated with increasing concentrations $(10,20,40,80$ and $160 \mu \mathrm{M})$ of oridonin for 24,48 and $72 \mathrm{~h}$, respectively. As presented in Fig. 3A, the inhibitory effects increased in a dose-dependent manner.

In order to determine whether oridonin exerts synergistic antitumor effects with cisplatin in ovarian cancer cells, $20 \mu \mathrm{M}$ was selected as the appropriate concentration for oridonin as the inhibition rate was effective at $~ 30 \%$ but relatively low. The concentrations of cisplatin used were 5, 10, 25, 50 and $100 \mu \mathrm{M}$. Compared with the group treated with oridonin alone, the inhibitory effects were significantly increased at $10,25(\mathrm{P}<0.05)$ and $50 \mu \mathrm{M}(\mathrm{P}<0.01)$, which demonstrated that oridonin and cisplatin demonstrate synergistic antitumor effects in ovarian cancer cells.

$I C_{50}$ values of cisplatin were decreased when administered in combination with $20 \mu \mathrm{M}$ oridonin in A2780/DDP and

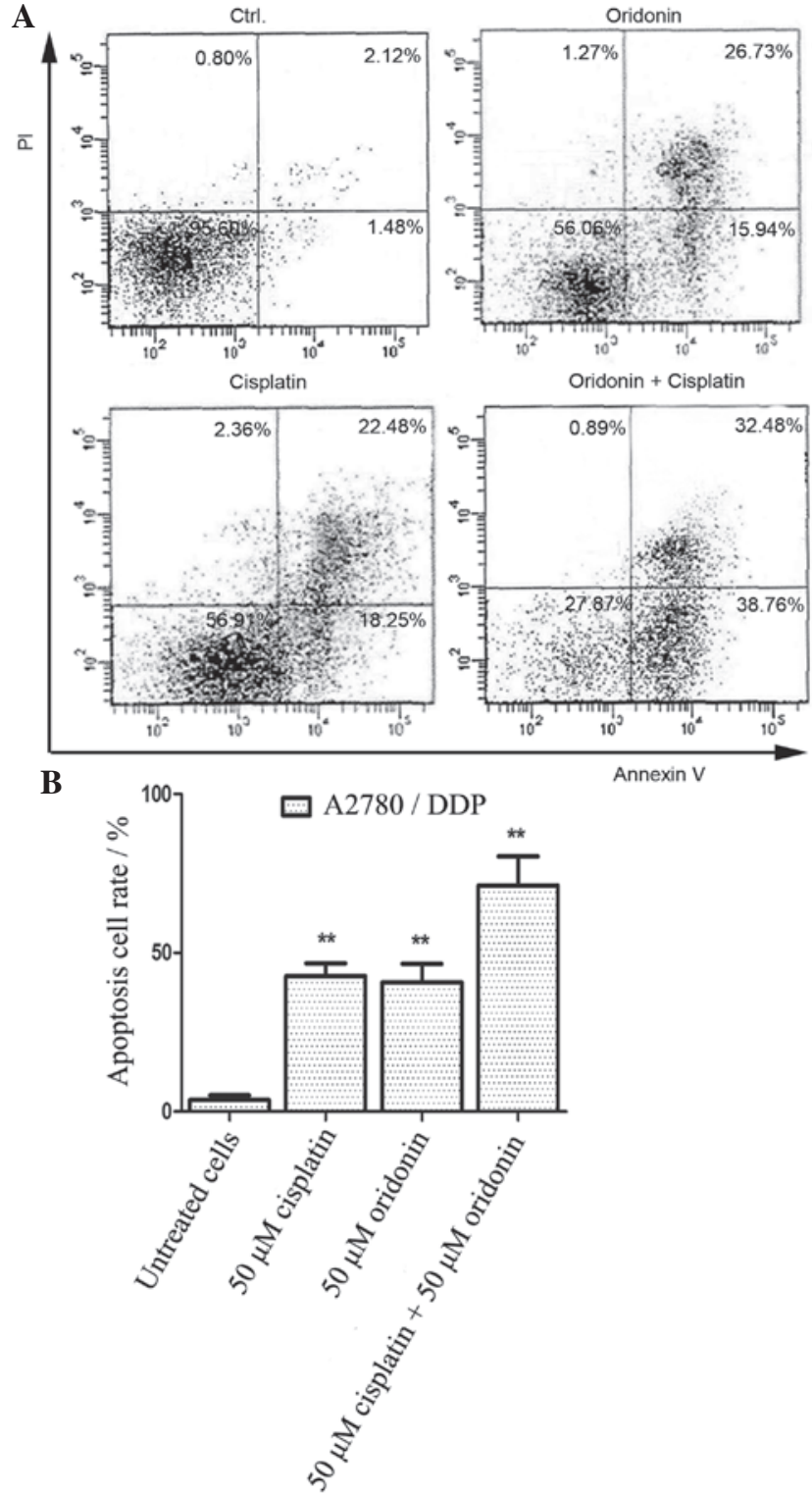

Figure 5. Cell apoptosis rates were detected by flow cytometry of A2780/DDP cells. A2780/DDP cells ( $2 \times 10^{4}$ cells/well) were plated into 6-well plates, cultured for 6 to $8 \mathrm{~h}$ and treated with cisplatin alone, oridonin alone, or cisplatin in combination with ordonin for $48 \mathrm{~h}$. Untreated cells served as negative controls. (A) Cell apoptosis rates were detected by fluorescence-activated cell sorting analysis with Annexin V and PI dual staining method. (B) The histogram of cell apoptosis rates is presented. ${ }^{* *} \mathrm{P}<0.01$ vs. the control. PI, propidium iodide.

SKOV3/DDP cells. Results from the present study demonstrated that oridonin synergistically increased the antitumor effects of cisplatin in A2780/DDP and SKOV3/DDP cells. The cells were treated with increasing concentrations of cisplatin in combination with $20 \mu \mathrm{M}$ oridonin for $48 \mathrm{~h}$. The samples had two replicates and the experiments were conducted twice. The untreated cells were used as negative controls. As presented in Fig. 4, the $\mathrm{IC}_{50}$ values were calculated as 26.12 and $73.00 \mu \mathrm{M}$ for $48 \mathrm{~h}$. The ratio of $\mathrm{IC}_{50}$ values was downregulated by $\sim 1.95$ and 1.84-fold in A2780/DDP and SKOV3/DDP cells treated with cisplatin $+20 \mu \mathrm{M}$ of oridonin, respectively, which demonstrated that oridonin significantly $(\mathrm{P}<0.01)$ decreased the resistance to cisplatin in A2780/DDP and SKOV3/DDP cells. 
A

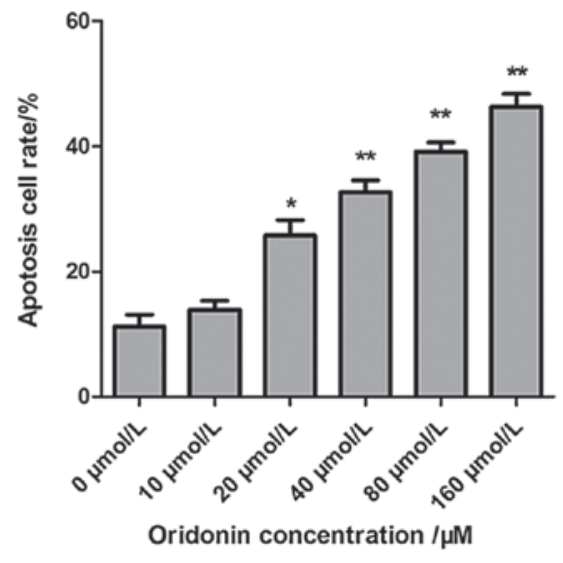

B

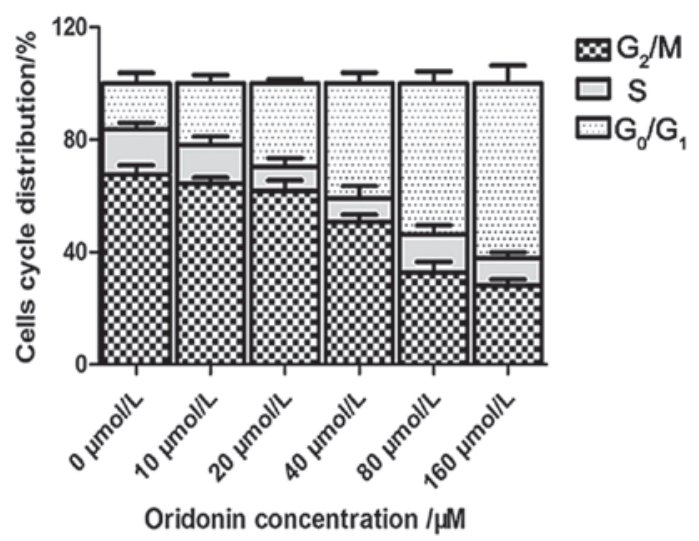

C Ctrl. Cisplatin Oridonin Cisplatin+Oridonin

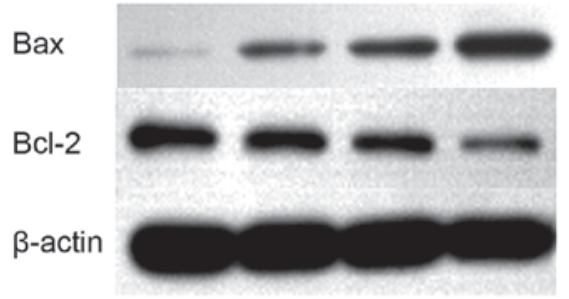

Figure 6. Cell apoptosis rate and the cell cycle distribution of A2780/DDP cells was determined. (A) A2780/DDP cells were treated with increasing concentrations of oridonin for $48 \mathrm{~h}$ with the untreated cells serving as negative controls. Cell apoptosis rate was determined by fluorescence-activated cell sorting analysis with Annexin V and PI dual staining. ${ }^{*} \mathrm{P}<0.05,{ }^{*} \mathrm{P}<0.01$ vs. the untreated cells. (B) Cell cycle of A2780/DDP cells was determined using the PI-staining method. A2780/DDP cells were treated with increasing concentrations of oridonin for $48 \mathrm{~h}$. The proportion of the cells in each phase was determined. The cell distribution is presented in the histogram. (C) Expression levels of apoptosis-associated proteins in A2780/DDP cells were detected by western blotting. A2780/DDP cells were treated with $20 \mu \mathrm{M}$ oridonin and $10 \mu \mathrm{M}$ cisplatin for $48 \mathrm{~h}$. Expression levels of Bax and Bcl-2 in A2780/DDP cells were detected by western blotting. The experiment was repeated 3 times. PI, propidium iodide.

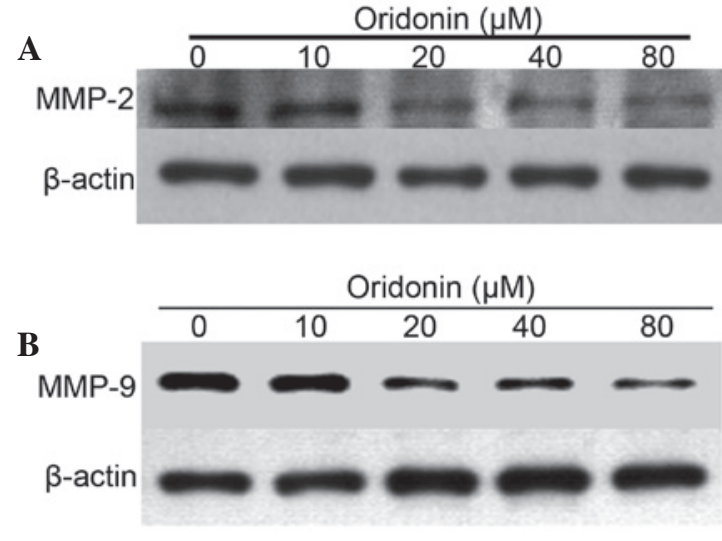

Figure 7. Expression levels of (A) MMP-2 and (B) MMP-9 in A2780/DDP cells were detected by western blot analysis. A2780/DDP cells were plated into 48 -well plates and cultured for $8 \mathrm{~h}$. The cells were treated with increasing concentrations of oridonin for $48 \mathrm{~h}$. Untreated cells served as negative controls. MMP, matrix metalloproteinase.

Oridonin and cisplatin synergistically induces cell apoptosis in A2780/DDP cells. The present study demonstrated that the cell death rates were significantly increased in the cisplatin and oridonin group. In order to identify whether cell apoptosis in A2780/DDP cells was induced by treatment with oridonin alone, cisplatin alone and oridonin in combination with cisplatin for $48 \mathrm{~h}$, fluorescence-activated cell sorting (FACS) analysis was performed to detect the cell apoptosis rate in
A2780/DDP cells. As presented in Fig. 5A, the cells were treated with $50 \mu \mathrm{M}$ cisplatin alone, $50 \mu \mathrm{M}$ oridonin alone, or $50 \mu \mathrm{M}$ cisplatin $+50 \mu \mathrm{M}$ oridonin for $48 \mathrm{~h}$, and the late apoptosis rate was $32.48 \%$ in the oridonin + cisplatin group, which was markedly higher than that of the oridonin or cisplatin alone groups. As presented in Fig. 5B, the early and late cell apoptosis rates were $42.67,40.73$ and $71.24 \%$ in the oridonin, cisplatin and oridonin + cisplatin groups, respectively. All the results demonstrated that oridonin and cisplatin act synergistically with cisplatin in inducing cell apoptosis in A2780/DDP cells.

Oridonin induces cell apoptosis of ovarian cancer cells and induces cell-cycle arrest at the $G_{0} / G_{1}$ phase. In order to further elucidate the underlying mechanism of cell apoptosis in cisplatin-resistant ovarian cancer cells, the A2780/DDP cells were treated with oridonin at 10, 20, 40, 80 and $160 \mu \mathrm{M}$ for $48 \mathrm{~h}$. As presented in Fig. 6A, the result demonstrated that oridonin induced cell apoptosis of A2780/DDP cells, which increased in a dose-dependent manner.

The cell cycle distribution of A2780/DDP cells was also analyzed by the PI-staining method. As presented in Fig. 6B, $\mathrm{G}_{0} / \mathrm{G}_{1}$ phase arrest of the cells was induced and the number of cells in $\mathrm{G}_{0} / \mathrm{G}_{1}$ phase increased in a dose-dependent manner, while the number of cells in $\mathrm{G}_{2} / \mathrm{M}$ phase decreased and the number in $\mathrm{S}$ phase was not markedly changed.

Oridonin and cisplatin synergistically downregulated the expression levels of Bcl-2 and upregulated the expression 
levels of Bax. The expression levels of Bcl-2 family proteins were detected by western blot analysis. As presented in Fig. 6C, treatment with oridonin resulted in downregulation of $\mathrm{Bcl}-2$ protein expression levels and the upregulation of Bax protein expression levels. This was consistent with cells that were treated with cisplatin alone. Notably, in A2780/DDP cells treated with $20 \mu \mathrm{M}$ oridonin and $10 \mu \mathrm{M}$ cisplatin for $48 \mathrm{~h}$, the ratio of Bax/Bcl-2 was markedly higher than that of cells treated with cisplatin or oridonin alone. All data indicated that oridonin and cisplatin synergistically downregulated the protein expression levels of $\mathrm{Bcl}-2$ and upregulated the protein expression levels of Bax.

Expression levels of MMP-2 and MMP-9 decreased in a dose-dependent manner with oridonin treatment. MMPs, including MMP-2 and MMP-9, are involved in the invasion and metastasis in a number of types of human malignancy, as degradation of collagen IV in the basement membrane and extracellular matrix facilitates tumor progression. The expression levels of MMP-2 and MMP-9 in A2780/DDP cells treated with an increasing concentration of oridonin were detected. A2780/DDP cells were treated with increasing concentrations $(10,20,40$ and $80 \mu \mathrm{M})$ of oridonin for $48 \mathrm{~h}$. As presented in Fig. 7, the protein expression levels of MMP-2 and MMP-9 decreased with increasing concentration of oridonin suggesting that oridonin may suppress the invasion and metastasis of human ovarian cancer cells.

\section{Discussion}

Adverse side-effects of chemotherapy and resistance to chemotherapeutic agents are a key problem in ovarian cancer therapy (26). Cisplatin resistance is a major obstacle in the treatment of ovarian cancer and novel chemotherapeutic strategies are urgently required $(4,27)$. The present study aimed to investigate a novel method to reverse cisplatin-resistance using combination therapy with oridonin and cisplatin in human ovarian cancer cells. The cisplatin-resistant A2780/DDP and SKOV3/DDP ovarian cancer cell lines were used as cell models. Results from the present study demonstrated that oridonin had a synergistic role with cisplatin to inhibit proliferation and induce cell apoptosis of cisplatin-resistant ovarian cancer cells.

The combined therapy of oridonin and cisplatin has a synergistic antitumor effect, which may decrease the dose required of a single therapeutic agent used. It is effective to kill tumor cells at a relatively lower dose in order to decrease the side effects of chemotherapeutic agents. The results demonstrated that cell death significantly increased in the cisplatin + oridonin group partly as a result of increased cell apoptosis. The apoptosis rate was $71.24 \%$ in the oridonin + cisplatin group, markedly higher than the rates of 42.67 and $40.73 \%$ in the oridonin and cisplatin groups, respectively. This was consistent with the detection of protein expression levels by western blot analysis. The downregulated protein expression level of Bcl-2 and upregulated protein expression level of Bax demonstrated that combination therapy with oridonin and cisplatin promoted cell apoptosis in drug-resistant A2780/DDP cells. Cell phase was detected by FACS, and the results demonstrated oridonin induces cell-cycle arrest in $\mathrm{G}_{0} / \mathrm{G}_{1}$ phase and the apoptosis rate increased in a dose-dependent manner with oridonin. All the results demonstrated that combined therapy is an effective method to inhibit the proliferation of human ovarian cancer cells.

Notably, the combined treatment of oridonin and cisplatin effectively reversed the cisplatin resistance. $\mathrm{The} \mathrm{IC}_{50}$ values were significantly decreased from 50.97 to $26.12 \mu \mathrm{M}$ in the A2780/DDP cells and, consistently, in the SKOV3/DDP cells, the $\mathrm{IC}_{50}$ values were decreased from 135.20 to $73.00 \mu \mathrm{M}$ at $48 \mathrm{~h}$.

MMPs are involved in the invasion and metastasis of human malignancies, and the present study demonstrated the expression levels of MMP-2 and MMP-9 were decreased in A2780/DDP cells treated with increasing concentration of oridonin. The data indicated that oridonin may suppress the invasion and metastasis of human ovarian cancer cells, which may be an effective therapeutic strategy to inhibit the spread of drug-resistant ovarian cancer cells.

In conclusion, combination therapy with oridonin and cisplatin was an useful method to treat cisplatin-resistant ovarian cancer cells. The two compounds exerted synergistic antitumor effects and effectively reversed the cisplatin resistance in human ovarian cancer cells.

\section{References}

1. Ozga M, Aghajanian C, Myers-Virtue S, McDonnell G, Jhanwar S, Hichenberg S and Sulimanoff I: A systematic review of ovarian cancer and fear of recurrence. Palliat Support Care 13: 1-10, 2015.

2. Ye H, Karim AA and Loh XJ: Current treatment options and drug delivery systems as potential therapeutic agents for ovarian cancer: A review. Mater Sci Eng C Mater Biol Appl 45: 609-619, 2014.

3. Zafrakas M, Grimbizis G, Timologou A and Tarlatzis BC: Endometriosis and ovarian cancer risk: A systematic review of epidemiological studies. Front Surg 1: 14, 2014.

4. Yoshida H, Teramae M, Yamauchi M, Fukuda T, Yasui T, Sumi T, Honda K and Ishiko O: Association of copper transporter expression with platinum resistance in epithelial ovarian cancer. Anticancer Res 33: 1409-1414, 2013.

5. Redman C, Duffy S and Dobson C: Improving early detection of ovarian cancer. Practitioner 255: 27-30, 2011.

6. Walters Haygood CL, Arend RC, Straughn JM and Buchsbaum DJ: Ovarian cancer stem cells: Can targeted therapy lead to improved progression-free survival? World J Stem Cells 6: 441-447, 2014.

7. Gallo D, Fruscella E, Ferlini C, Apollonio P, Mancuso S and Scambia G: Preclinical in vivo activity of a combination gemcitabine/liposomal doxorubicin against cisplatin-resistant human ovarian cancer (A2780/CDDP). Int J Gynecol Cancer 16: 222-230, 2006.

8. Song T, Kim MK, Lee YY, Choi CH, Kim TJ, Lee JW, Kim BG and Bae DS: Phase II study of ifosfamide and cisplatin for the treatment of recurrent ovarian cancer. Cancer Chemother Pharmacol 72: 653-660, 2013.

9. Musiani D, Hammond DE, Cirillo L, Erriquez J, Olivero M, Clague MJ and Di Renzo MF: PIM2 kinase is induced by cisplatin in ovarian cancer cells and limits drug efficacy. J Proteome Res 13: 4970-4982, 2014

10. Ziyan W and Yang L: MicroRNA-21 regulates the sensitivity to cisplatin in a human osteosarcoma cell line. Ir J Med Sci 185: 85-91, 2014

11. Lockhart AC, Sundaram S, Sarantopoulos J, Mita MM, Wang-Gillam A, Moseley JL, Barber SL, Lane AR, Wack C, Kassalow L, et al: Phase I dose-escalation study of cabazitaxel administered in combination with cisplatin in patients with advanced solid tumors. Invest New Drugs 32: 1236-1245, 2014.

12. Costello BA, Borad MJ, Qi Y, Kim JP, Northfelt DW, Erlichman C and Alberts SR: Phase I trial of everolimus, gemcitabine and cisplatin in patients with solid tumors. Inves New Drugs 32: 710-716, 2014. 
13. Joo WD, Lee JY, Kim JH, Yoo HJ, Roh HJ, Park JY, Kim DY, Kim YM, Kim YT and Nam JH: Efficacy of taxane and platinum-based chemotherapy guided by extreme drug resistance assay in patients with epithelial ovarian cancer. J Gynecol Oncol 20: 96-100, 2009.

14. Kim HS, Kim TJ, Chung HH, Kim JW, Kim BG, Park NH, Song YS, Bae DS and Kang SB: In vitro extreme drug resistance assay to taxanes or platinum compounds for the prediction of clinical outcomes in epithelial ovarian cancer: A prospective cohort study. J Cancer Res Clin Oncol 135: 1513-1520, 2009.

15. Karam AK, Chiang JW, Fung E, Nossov V and Karlan BY: Extreme drug resistance assay results do not influence survival in women with epithelial ovarian cancer. Gynecol Oncol 114 246-252, 2009.

16. Zhang H, Wang J, Cai K, Jiang L, Zhou D, Yang C, Chen J, Chen D and Dou J: Downregulation of gene MDR1 by shRNA to reverse multidrug-resistance of ovarian cancer A2780 cells. J Cancer Res Ther 8: 226-231, 2012.

17. Wang S, Zhong Z, Wan J, Tan W, Wu G, Chen M and Wang Y: Oridonin induces apoptosis, inhibits migration and invasion on highly-metastatic human breast cancer cells. Am J Chin Med 41: 177-196, 2013.

18. Gao FH, Hu XH, Li W, Liu H, Zhang YJ, Guo ZY, Xu MH, Wang ST, Jiang B, Liu F, et al: Oridonin induces apoptosis and senescence in colorectal cancer cells by increasing histone hyperacetylation and regulation of p16, p21, p27 and c-myc. BMC Cancer 10: 610, 2010.

19. Qi X, Zhang D, Xu X, Feng F, Ren G, Chu Q, Zhang Q and Tian K: Oridonin nanosuspension was more effective than free oridonin on $\mathrm{G} 2 / \mathrm{M}$ cell cycle arrest and apoptosis in the human pancreatic cancer PANC-1 cell line. Int J Nanomedicine 7: 1793-1804, 2012.
20. Gao FH, Liu F, Wei W, Liu LB, Xu MH, Guo ZY, Li W, Jiang B and $\mathrm{Wu}$ YL: Oridonin induces apoptosis and senescence by increasing hydrogen peroxide and glutathione depletion in colorectal cancer cells. Int J Mol Med 29: 649-655, 2012.

21. Cui Q, Tashiro S, Onodera S, Minami M and Ikejima T: Autophagy preceded apoptosis in oridonin-treated human breast cancer MCF-7 cells. Biol Pharm Bull 30: 859-864, 2007.

22. Wang YY, Lv YF, Lu L and Cai L: Oridonin inhibits mTOR signaling and the growth of lung cancer tumors. Anticancer Drugs 25: 1192-1200, 2014

23. Ding Y, Wang Y, Chen J, Hu Y, Cao Z, Ren P and Zhang Y: P21 overexpression sensitizes osteosarcoma U2OS cells to cisplatin via evoking caspase-3 and $\mathrm{Bax} / \mathrm{Bcl}-2$ cascade. Tumour Biol 35: 3119-3123, 2014.

24. Hoshyar R, Bathaie SZ and Sadeghizadeh M: Crocin triggers the apoptosis through increasing the $\mathrm{Bax} / \mathrm{Bcl}-2$ ratio and caspase activation in human gastric adenocarcinoma, AGS, cells. DNA Cell Biol 32: 50-57, 2013

25. Lu HF, Chie YJ, Yang MS, Lee CS, Fu JJ, Yang JS, Tan TW, Wu SH, Ma YS, Ip SW and Chung JG: Apigenin induces caspase-dependent apoptosis in human lung cancer A549 cells through Bax- and Bcl-2-triggered mitochondrial pathway. Int J Oncol 36: 1477-1484, 2010

26. Huq F, Yu JQ, Beale P, Chan C, Arzuman L, Nessa MU and Mazumder ME: Combinations of platinums and selected phytochemicals as a means of overcoming resistance in ovarian cancer. Anticancer Res 34: 541-545, 2014.

27. Gamarra-Luques CD, Hapon MB, Goyeneche AA and Telleria CM: Resistance to cisplatin and paclitaxel does not affect the sensitivity of human ovarian cancer cells to antiprogestin-induced cytotoxicity. J Ovarian Res 7: 45, 2014. 